\title{
Isolation, identification of an aluminum-tolerant strain and its tolerance
}

\author{
characteristics \\ Yi Li ${ }^{1,2, a *}$, Wentao Peng ${ }^{1, b}$, Qinfen $\mathrm{Li}^{1,2, \mathrm{c}}$, Wei $\mathrm{Li}^{1,2, \mathrm{~d}}$ \\ ${ }^{1}$ Environment and Plant Protection Institute, Chinese Academy of Tropical Agricultural Sciences, \\ Haikou, Hainan 571101, China \\ ${ }^{2}$ Danzhou Scientific Observing and Experimental Station of Agro-Environment, Ministry of \\ Agriculture, Danzhou, Hainan 571737, China \\ awish.0310@163.com, bdawentao@163.com, ㅁinfenli2005@163.com, d530243942@qq.com, \\ ${ }^{*}$ Corresponding author
}

\section{Keywords: Aluminum tolerance; Bacteria; Tolerance characteristics}

Abstract. In recent years, aluminum enrichment in soils is exacerbated. The amount of aluminum exchange in acidic soils directly affects the level of soil acidification. Aluminum toxicity-neutralizing capabilities of the microorganisms that have survived under the aluminum toxicity selection determine the harmfulness of aluminum toxicity in soils. Aluminum-tolerant microorganisms, especially aluminum-tolerant bacteria, are ideal for the study of aluminum-tolerance mechanisms. There are not many types of aluminum-tolerant bacteria. In acid soils, fungi are more tolerant to aluminum than bacteria. In addition, the isolation of aluminum-tolerant bacteria is more difficult. Thus, the mechanistic study of aluminum tolerance progressed very slowly. In this study, we isolated a bacteria strain A1 that is highly aluminum tolerant. It tolerated $\mathrm{Al}^{3+}$ at the concentration of $10 \mathrm{mmol} / \mathrm{L}$. This strain was identified as Citrobacter sp., and its capability and features of aluminum tolerance were investigated. Our study provided resource and theoretical basis for the application of aluminum-tolerant bacteria in soils.

\section{Introduction}

In acid soils, the amount of aluminum exchange directly affects the level of soil acidification. With the decrease of soil $\mathrm{pH}$, active aluminum is released into the soil solution as ions, which exert a great toxic effect on organisms in soils [1]. In recent years, aluminum enrichment in soils has been exacerbating and the amount of active aluminum in the environment has increased substantially [2].

Soil microorganisms contain nutrients with high availability. They promote material cycling and energy exchange in the soil ecological system, and play important roles in determining the soil quality and fertility. Therefore, the loss of microorganisms due to the exacerbated aluminum toxicity has led to soil acidification and the fragile ecological system.

Microorganisms are very sensitive to environmental changes and can serve as the early indicators for the functional changes of the ecological system. Therefore, they play an indispensable role in the bio-restoration process of the soils. Microorganisms commonly grow fast, with short doubling time and high mutation rate. Aluminum toxicity-neutralizing capabilities of the microorganisms that have survived under the aluminum toxicity selection determine the harmfulness of aluminum toxicity in soils. A few new methods could be applied to alleviate aluminum toxicity and to improve the soil quality, including absorption and adsorption by aluminum-tolerant microorganisms, or aluminum ion chelation by metabolites secreted by these microorganisms.. Aluminum-tolerant microorganisms, especially aluminum-tolerant bacteria, are ideal for the study of aluminum-tolerance mechanisms.

A number of aluminum-tolerant microorganisms have been isolated and reported. These 
aluminum-tolerant bacteria include Acidocella aluminiidurans, Flavobacterium sp., Pseudomonas fluorescens, Arthrobacter sp., Burkholderia sp., and Bacillus cereus B5 [3-9]. The reported aluminum-tolerant fungi include Saccharomyces cerevisiae, Lipomyces, Rhodotorula taiwanensis RS1 [9-11]. At present, most of the obtained fungi are yeasts. Very few types of aluminum-tolerant bacteria have been obtained, which are mainly Pseudomonas fluorescens and Burkholderia sp. The reason is that in acidic soils, fungi are more tolerant to aluminum than bacteria, and the isolation of aluminum-tolerant bacteria is more difficult [12]. Thus, the mechanistic study of aluminum tolerance progressed slowly. In this study, we isolated a bacteria strain A1 that is highly aluminum tolerant, and investigated its capability and features of aluminum tolerance. Our study provided resource and theoretical basis for the application of aluminum-tolerant bacteria in soils.

\section{Methods}

Strain isolation. An acidic LB medium with the $\mathrm{pH}$ value of 5 (adjusted by using $\mathrm{HCl}$ ) and the $\mathrm{Al}^{3+}$ concentration of $5 \mathrm{mmol} / \mathrm{L}$ (aluminum sulfate) was prepared for the isolation of acid- and aluminum-tolerant bacterial strain. One gram of fresh soil sample was added to $10 \mathrm{~mL}$ culture medium and the medium was cultured overnight on a shaker. The diluted medium was then spread on LB plates and cultured for 3-7 days at $28^{\circ} \mathrm{C}$, until colonies were visible. An inoculating needle was used to pick a colony and to streak on another LB plate for purification. Plate streaking was performed 3 times until single colonies were observed, and a pure culture of the microorganism was confirmed.

Strain identification. The genomic DNA was extracted from the A1 strain and 16S rDNA was amplified to be analyzed at the NCBI website (https://blast.ncbi.nlm.nih.gov/Blast.cgi). The sequences with high homology were used to construct the phylogenetic tree.

Evaluating aluminum-tolerant capability. A single colony was picked and cultured in liquid LB medium overnight. When the $\mathrm{A}_{600}$ reached 1.0, the culture medium was centrifuged, and the pellet was washed twice using sterile distilled water and then resuspended in $1 \mathrm{~mL}$ sterile distilled water. The suspension was diluted using sterile distilled water until its $\mathrm{A}_{600}$ value reached 1.0. The suspension was then diluted 10 times or 100 times and plated on solid LB plates containing different concentrations of aluminum. The plates were cultured at $30{ }^{\circ} \mathrm{C}$ for 3 days and an aluminum-sensitive bacterium was used as the control.

Growth curve of the strain. A bacterial culture $(1 \mathrm{~mL})$ with the $\mathrm{A}_{600}$ value of 1.0 was inoculated to $20 \mathrm{~mL} \mathrm{LB}$ medium containing $5 \mathrm{mmol} / \mathrm{L}$ aluminum. The LB medium without aluminum was used as the control. Growth curve was determined.

\section{Results and Discussions}

The separation and identification of the strain. An aluminum-tolerant bacterial strain was isolated after screening from acid soils and was named as A1. Its 16S rDNA sequence was analyzed using BLAST on NCBI and a phylogenetic tree was constructed accordingly (Figure. 1).

As shown in Fig. 1, the A1 strain was highly homologous with Citrobacter sp. and exhibited the closest homology with Citrobacter europaeus (LT615140). Their 16S rDNA homology was 99.92\%. In addition, according to the phylogenetic tree, the A1 strain was on the same sub-branch with other strains including Citrobacter braakii, Citrobacter murliniae, Citrobacter portucalensis, and Citrobacter werkmanii. Therefore, the strain A1 was identified as Citrobacter sp.. 


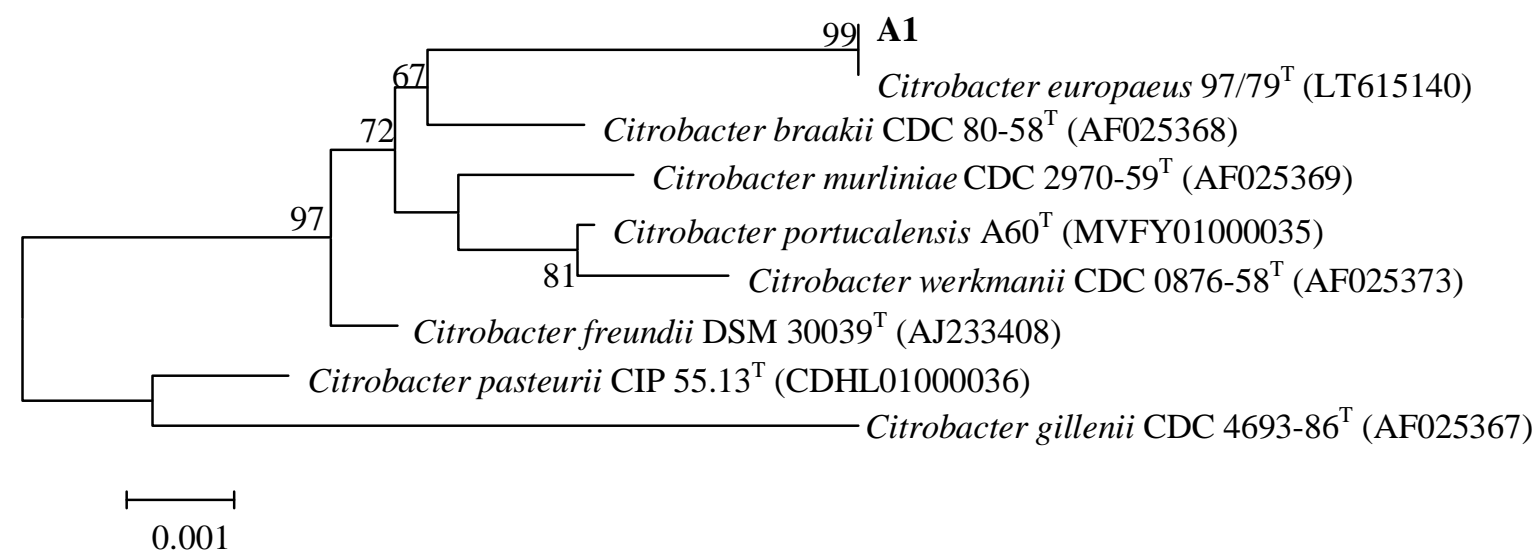

Fig. 1 Phylogenetic tree of strain A1 based on 16S rRNA gene. Distances were calculated and clustering with the neighbour-joining method performed by using the software package MEGA version 5.0. Numbers at nodes are percentage bootstrap values based on 1000 resampled datasets; only values above $60 \%$ are given. Bar, 0.001 substitutions per nucleotide position.

Evaluating aluminum-tolerant capability. Different amount of bacteria from the control strain or the A1 strain were inoculated to $\mathrm{LB}$ medium containing various concentrations of $\mathrm{Al}^{3+}$. The results were shown in Table 1.

Table 1 Growth condition of the A1 strain in LB culture medium containing different concentrations of $\mathrm{Al}^{3+}$ ("+" indicates growth and "-" indicates no growth)

\begin{tabular}{ccccccccccccccc}
\hline $\mathrm{c}\left(\mathrm{Al}^{3+}\right)[\mathrm{mmol} / \mathrm{L}]$ & & 0 & \multicolumn{1}{c}{5} & \multicolumn{1}{c}{10} & & & & & & & \\
\hline $\mathrm{A}_{600}$ & 1 & 0.1 & 0.01 & 1 & 0.1 & 0.01 & 1 & 0.1 & 0.01 & 1 & 0.1 & 0.01 \\
\hline $\mathrm{CK}$ & + & + & + & - & - & - & - & - & - & - & - & - \\
Strain A1 & + & + & + & + & + & + & + & + & + & - & - & - \\
\hline
\end{tabular}

As shown in Table 1, the A1 strain grew in medium containing 0-10 $\mathrm{mmol} / \mathrm{L}$ aluminum, while the control strain only grew in the aluminum-free medium. This indicates that the A1 strain is more aluminum tolerant compared to the control strain, with its maximum tolerant level at $10 \mathrm{mmol} / \mathrm{L}$.

Growth curve of the strain. The A1 strain growth curves in the $\mathrm{Al}^{3+}$-free medium and the medium containing $5 \mathrm{mmol} / \mathrm{L} \mathrm{Al}^{3+}$ were measured. The results were shown in Fig. 2.

As shown in Fig. 2, at 24 hours after culture, $\mathrm{Al}^{3+}$ did not affect the growth. However, after 24 hours, $\mathrm{Al}^{3+}$ significantly impaired the growth of the A1 strain. This is possibly due to the toxicity of $\mathrm{Al}^{3+}$, by which the growth of the $\mathrm{A} 1$ was inhibited and the proliferation rate was subsequently decreased. 


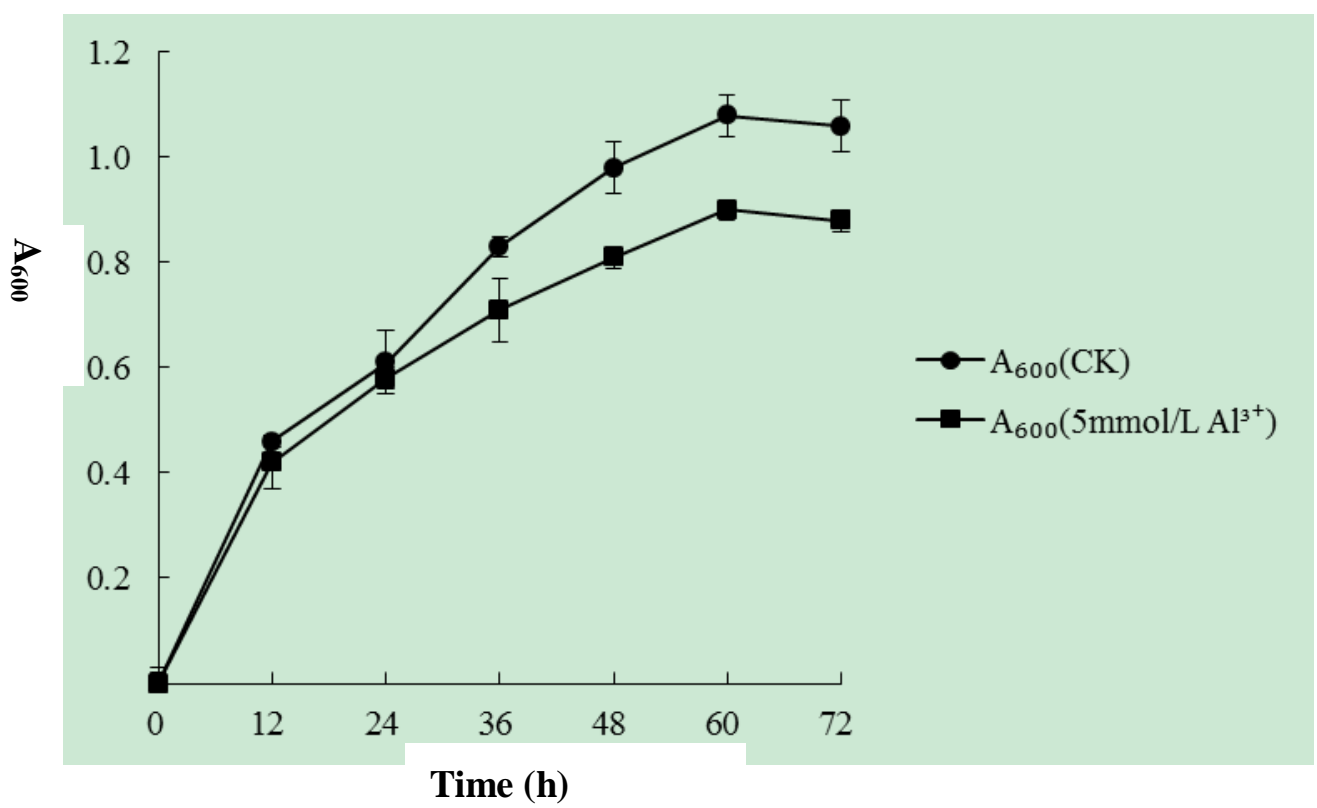

Fig. 2 The growth curve of the $\mathrm{A} 1$ strain in the culture medium without $\mathrm{Al}^{3+}$ or with $5 \mathrm{mmol} /{\mathrm{L} \mathrm{of} \mathrm{Al}^{3+}}^{3+}$

\section{Conclusions}

From acid soils, we isolated a bacteria strain A1 that is highly aluminum tolerant. This strain was identified as Citrobacter sp. and it tolerated $\mathrm{Al}^{3+}$ up to the concentration of $10 \mathrm{mmol} / \mathrm{L}$. However, with the presence of $\mathrm{Al}^{3+}$, the growth of the bacteria was inhibited at the later stage.

\section{Acknowledgements}

This work was financially supported by the National Natural Science Foundation of China (41701307), Natural Science Foundation of Hainan Province (20164169), the Central Public-interest Scientific Institution Basal Research Fund (NO. 1630042017034) and the Central Public-interest Scientific Institution Basal Research Fund (NO. 1630022017009).

\section{References}

[1] T. Eekhout, P. Larsen and L.De. Veylder: Trends. Plant. Sci Vol. 22 (2017), p. 102

[2] H. Sade, B. Meriga, V. Surapu, J. Gadi, M.S.L. Sunita, P. Suravajhala and P.B.K. Kishor: Biometals Vol. 29 (2016), p. 187

[3] B. Ji, W. Chen, L. Zhu and K. Yang: Mar. Pollut. Bull Vol. 106 (2016), p. 31

[4] C. Auger, S. Han, V.P. Appanna, S.C. Thomas, G. Ulibarri and V.D. Appanna: Biotechnol. Adv Vol. 31 (2013), p. 266

[5] K. Kimoto, T. Aizawa, M. Urai, N.B.Ve, K.I. Suzuki, M. Nakajima and M. Sunairi: Int. J. Syst. Evol. Microbiol Vol. 60 (2010), p. 764

[6] S. Konishi, I. Souta, J. Takahashi, M. Ohmoto and S. Kaneko: Biosci. Biotechnol. Biochem Vol. 58 (1994), p. 1960

[7] J. Lemire, R. Mailloux, C. Auger, D. Whalen and V.D. Appanna: Environ. Microbiol Vol. 12 (2010), p. 1384

[8] P. Illmer and C. Erlebach: Anton. Leeuw Vol. 84 (2003), p. 239

[9] T. Kunito, M. Owaki, Y. Ihyo, H. Sumi, H. Toda, D. Fukuda and H.D. Park: Ann. Microbiol Vol. 62 (2012), p. 1339 
[10] C.A. Hamilton, A.G. Good and G.J. Taylor: FEMS Microbiol. Lett Vol. 205 (2001), p. 231

[11] Z.M. Hu, X.Q. Zhao, X.M. Bao, C. Wang, W. Wang, L. Zheng, P. Lan and R.F. Shen: Yeast Vol. 33 (2016), p. 575

[12] S. Kanazawa, T. Chau, N. Thi and S. Miyaki: Soil. Sci. Plant. Nutr Vol. 51 (2005), p. 507 